\title{
Efficient Charge Transport Enables High Efficiency in Dilute Donor Organic Solar Cells
}

\author{
Nannan Yao, Jianqiu Wang, Zeng Chen, Qingzhen Bian, Yuxin Xia, Rui Zhang, Jianqi Zhang, \\ Leiqiang Qin, Haiming Zhu, ${ }^{*}$ Yuan Zhang,* and Fengling Zhang*
}

Cite This: J. Phys. Chem. Lett. 2021, 12, 5039-5044

Read Online

\section{ACCESS | Lill Metrics \& More | 回 Article Recommendations ｜（） Supporting Information}

ABSTRACT: The donor/acceptor weight ratio is crucial for photovoltaic performance of organic solar cells (OSCs). Here, we systematically investigate the photovoltaic behaviors of PM6:Y6 solar cells with different stoichiometries. It is found that the photovoltaic performance is tolerant to PM6 contents ranging from 10 to $60 \mathrm{wt} \%$. Especially an impressive efficiency over $10 \%$ has been achieved in dilute donor solar cells with 10 wt \% PM6 enabled by efficient charge generation, electron/ hole transport, slow charge recombination, and field-insensitive extraction. This raises the question about the origin of efficient hole transport in such dilute donor structure. By investigating hole mobilities of PM6 diluted in Y6 and insulators, we find that effective hole transport pathway is mainly through PM6 phase in PM6:Y6 blends despite with low PM6 content. The results indicate that a low fraction of polymer donors combines with near-infrared nonfullerene acceptors could achieve high photovoltaic performance, which might be a candidate for semitransparent windows.

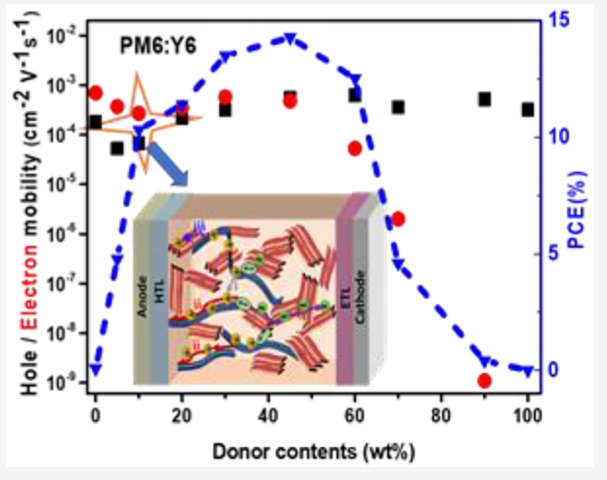

$\mathrm{O}$ rganic solar cells (OSCs) based on nonfullerene acceptors (NFAs) have achieved rapid development in recent years due to the tunable energy levels and absorption spectra of NFAs. ${ }^{1-6}$ The power conversion efficiency (PCE) of single-junction OSCs has reached over $18 \% .^{7-9}$ In NFA-OSCs, efficient charge separation and low voltage losses can be achieved simultaneously, yielding high short circuit current density $\left(J_{s c}\right)$ and open-circuit voltage $\left(V_{\text {oc }}\right) \cdot{ }^{10-12}$ The third important parameter fill factor (FF) is strongly affected by the competition between charge transport and recombination. It has been demonstrated that efficient charge transport and suppressed recombination losses are largely related to balanced electron and hole mobility in OSCs. ${ }^{13,14}$ Generally, a continuous interpenetrating network of donor and acceptor is considered to be significant for charge transport. ${ }^{15}$ Therefore, the donor/acceptor (D/A) blend composition is important for charge transport and device performance. Typically, in dilute donor heterojunctions where the average distance between individual donor domain enlarges, that may reduce hopping rate of hole. The unbalanced charge transport can induce a space-charge effect, causing more recombination loss and poor performance. ${ }^{16}$ Interestingly, some previous studies have reported that fullerene based OSCs with very low donor contents could maintain efficient hole transport. ${ }^{17,18}$ It was proposed that hole tunneling could occur between isolated donors, ${ }^{19,20}$ another possible channel is that fullerene acceptors such as PCBM can act as an ambipolar conductor for both electrons and holes in dilute donor solar cells. ${ }^{17,21,22}$

Compared to the clarification on the exclusive role of fullerene acceptors in dilute donor solar cells, charge generation, transport, and recombination in dilute donor devices with NFAs are rarely investigated. Recently, NFA Y6 with the $\mathrm{A}-\mathrm{D}-\mathrm{A}^{\prime}-\mathrm{D}-\mathrm{A}$ configuration has received great attention. In the optimized solar cells based on PM6:Y6 (1:1.2, $\mathrm{w} / \mathrm{w})$, a high $J_{\mathrm{sc}}$ exceeding $25 \mathrm{~mA} \mathrm{~cm}^{-2}$ with a low voltage loss $(\sim 0.48 \mathrm{eV})$ can be achieved simultaneously. ${ }^{6,23}$ A report with single crystal analysis and molecular simulation has highlighted the unique packing in $\mathrm{Y} 6$ films can lead to strong electronic coupling between adjacent Y6 molecules and efficient 3dimensional ambipolar transport networks. ${ }^{24}$ These interesting phenomena raise an important question about hole transport mechanisms in bulk heterojunctions ( $\mathrm{BHJs}$ ) with this emerging family of NFAs, which could contribute to high device performance. Dilute donor solar cells are interesting models to understand the role of acceptors on charge generation, transport and extraction. Therefore, it will be informative to investigate the correlation between photovoltaic performance and donor/acceptor blend composition in NFA-OSCs.

In this work, we studied the photovoltaic behaviors of PM6:Y6 solar cells with various PM6 contents. The OSCs with 10 wt \% PM6 yield a high PCE of $10.3 \%$ with $J_{\text {sc }}$ of $18.5 \mathrm{~mA}$ $\mathrm{cm}^{-2}$ and FF of 0.66 . The results of femtosecond transient

Received: April 15, 2021

Accepted: May 20, 2021

Published: May 21, 2021 
absorption (TA) spectroscopy indicate efficient charge transfer in dilute donor blend films. Moreover, slow charge recombination and almost field-independent charge extraction in the dilute donor devices were observed in dilute donor solar cells. Importantly, it is found that PM6:Y6 blends even with 10 wt \% PM6 exhibit efficient hole mobility and balanced charge transport. By comparing the hole mobility of PM6 diluted at 10 wt \% in Y6 and insulating polymers, we conclude that $10 \mathrm{wt}$ \% PM6 in PM6:Y6 blends can still retain efficient hole transport pathway through the active layer.

To study the photovoltaic performance, PM6:Y6 solar cells with various PM6 contents were fabricated with a standard structure of ITO/PEDOT:PSS/active layer/PDINO/Al. The photovoltaic parameters extracted from $J-V$ curves (Figure S1) are summarized in Figure 1 and Table S1. As shown in

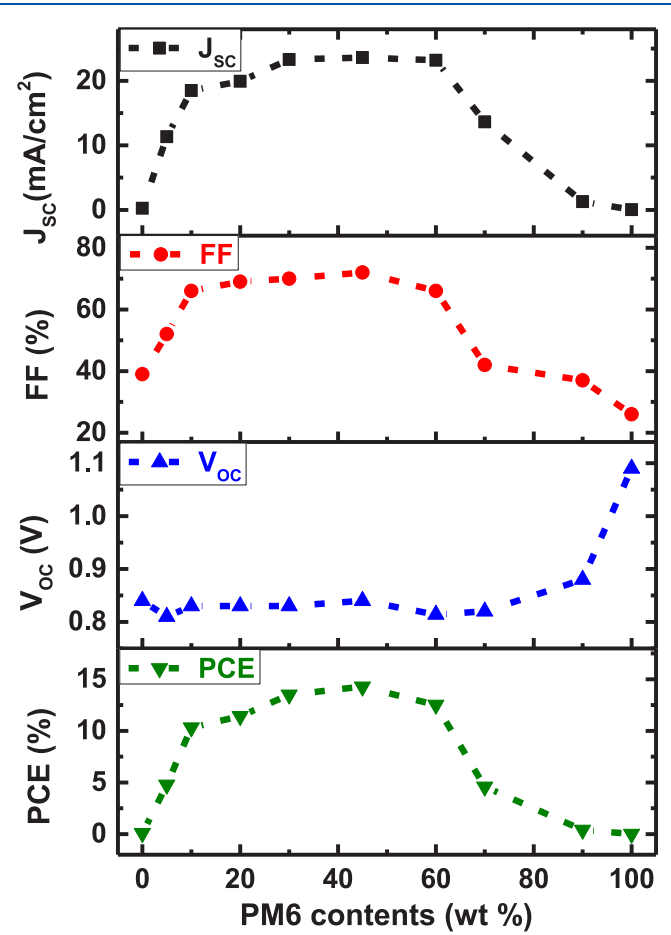

Figure 1. Correlation between photovoltaic performance of PM6:Y6 solar cells and PM6 contents (under $100 \mathrm{~mW} \mathrm{~cm}^{-2}$ solar illumination). The dashed lines are guides to the eyes.
Figure 1, the performance of the OSCs exhibit a high tolerance to donor content ranging from 10 to $60 \mathrm{wt} \%$. OSCs with 10 wt\% PM6 yield an efficiency over $10 \%$ with a high a $J_{s c}$ of 18.5 $\mathrm{mA} \mathrm{cm}{ }^{-2}$ and $\mathrm{FF}$ of 0.66 . It is worth noting that dilute donor devices show much better performance than the devices with high donor contents, especially $J_{\mathrm{sc}}$ and FF. To understand the origin of high performance of the OSCs with such low donor contents, charge generation, transport, and recombination were investigated.

Charge transfer between PM6 and Y6 was investigated by measuring steady-state photoluminescence (PL) spectroscopy (Figure S2). Compared to the PL of pure Y6 films, the large degree of PL quenching in 10 wt \% PM6 $\mathrm{BHJ}$ indicates efficient interfacial charge transfer. Hole transfer dynamics from Y6 to PM6 was studied with femtosecond TA spectroscopy. In Figure S3 and Figure S4, a $750 \mathrm{~nm}$ pump laser was used to selectively excited Y6. The rising kinetics of PM6 ground state bleach in the blend at $\sim 605 \mathrm{~nm}$ reflects the hole transfer process from Y6 to PM6, since photoexcited Y6 shows no TA signal there (Figure S3). The hole transfer process exhibits a biphasic behavior with an ultrafast interfacial hole transfer process and a relatively slower diffusion mediated process. ${ }^{25-27}$ We fit the hole transfer kinetics (Table S2) by a biexponential function

$$
I=A_{1} \exp \left(-\frac{t}{\tau_{1}}\right)+A_{2} \exp \left(-\frac{t}{\tau_{2}}\right)
$$

with two lifetimes of $\tau_{1}$ and $\tau_{2}$ and prefactors of $A_{1}$ and $A_{2}{ }^{28}$ The initial ultrafast charge transfer process (Figure 2a) indicated by $\tau_{1}(<250 \mathrm{fs})$ can be attributed to quantum coherence, which plays a role in the formation of charge carriers $^{29}$ and was experimentally observed in OSCs. ${ }^{30}$ The exciton diffusion process characterized by $A_{2}$ and $\tau_{2}$ becomes more dominant with the decreasing of PM6 contents. Typically, large amounts of small molecules tend to aggregate, ${ }^{25}$ there would be expected larger domains in 10 wt \% PM6 BHJ films. The larger root-mean-square (RMS) of 10 wt \% PM6:Y6 films (Figure S5) also indicates strong molecular aggregation, which could lead to oversized domains. $^{31}$ Our previous work has shown that excitons will be created away from the interface and thus spend longer time to diffuse to the interfaces because of the larger domain in $\mathrm{BHJ}$ films with low donor contents. ${ }^{25}$ Similarly, the exciton diffusion
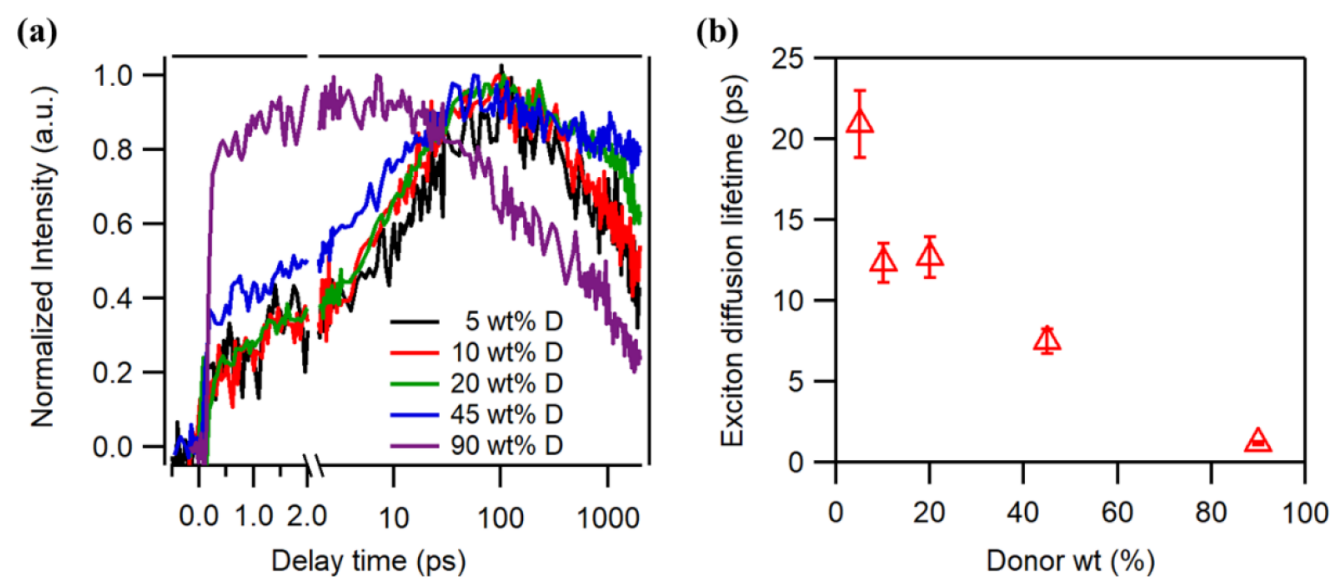

Figure 2. (a) Normalized TA kinetics of hole transfer in PM6:Y6 blend films with various PM6 contents (pump at $750 \mathrm{~nm}$, probe at $605 \mathrm{~nm}$ ). (b) Exciton diffusion lifetime as a function of PM6 contents (wt \%). 
mediated hole transfer lifetime $\tau_{2}$ in PM6:Y6 blends decreases with the amount of donor, as shown in Figure 2b. Here, we also calculated the hole transfer efficiency (HTE) of PM6:Y6 BHJs, as shown in Table S3. A THE of $83 \%$ in 10 wt \% PM6:Y6 blend reveals efficient hole transfer. In addition, the decay signals in Figure 2a indicate that PM6:Y6 blend films with low donor contents behave slower decay (half-lifetime of 1.45 ns for 5\% PM6-based BHJs and 1.5 ns for 10 wt \% PM6based BHJs) than 90 wt \% PM6-based BHJs ( 0.35 ns), suggesting slower charge recombination in dilute donor blends. In OSCs, if the charge transport is not efficient, charge recombination will occur after exciton dissociation.

To investigate the charge transport, charge carrier mobilities were determined from space charge limited current (SCLC) in single-carrier devices, as shown in Figure S6 and Figure 3.

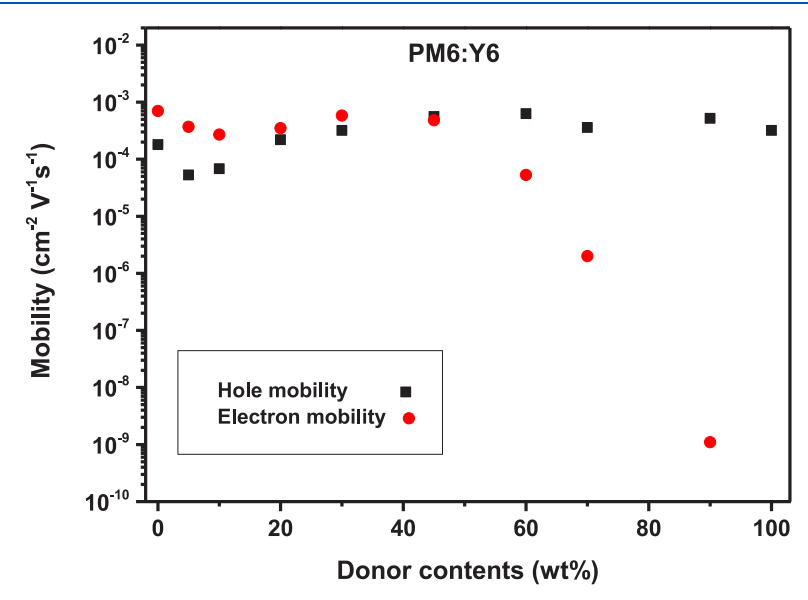

Figure 3. Electron and hole mobilities in PM6:Y6 OSCs with various donor contents extracted from SCLC in single-carrier devices.
Interestingly, the hole mobilities have little correlation with the PM6 contents, the devices even with 10 wt \% PM6 can still exhibit a high electron mobility of $2.7 \times 10^{-4} \mathrm{~cm}^{2} \mathrm{~V}^{-1} \mathrm{~s}^{-1}$ and a hole mobility of $6.8 \times 10^{-5} \mathrm{~cm}^{2} \mathrm{~V}^{-1} \mathrm{~s}^{-1}$ (Table S4), indicating efficient charge transport in such dilute donor structure. However, the electron mobilities show an obvious decrease when PM6 contents is over $60 \mathrm{wt} \%$, the unbalanced charge transport results in a decreased device performance (Figure 1). It is worth noting that pristine Y6 devices show both high electron mobility $\left(6.5 \times 10^{-4} \mathrm{~cm}^{2} \mathrm{~V}^{-1} \mathrm{~s}^{-1}\right)$ and hole mobility $\left(1.8 \times 10^{-4} \mathrm{~cm}^{2} \mathrm{~V}^{-1} \mathrm{~s}^{-1}\right)$, indicating $\mathrm{Y} 6$ is an ambipolar conductor for electron and hole.

To study the charge recombination in device operational conditions, we examined $J_{\text {sc }}$ under different light intensities. As shown in Figure 4a, the calculated slopes $(\alpha)$ for 10 and $45 \mathrm{wt}$ $\%$ PM6 based solar cells are 0.94 and 0.95 , indicating smaller bimolecular recombination loss. The relatively low $\alpha=0.76$ in the device based on 90 wt \% PM6 implies stronger recombination, which indicates that there is the emergence of strong space charges effect in $90 \mathrm{wt} \%$ donor devices mainly caused by extremely unbalanced charge transport. ${ }^{16,32}$ Figure $4 \mathrm{~b}$ shows $V_{\text {oc }}$ dependence on light intensity, the slopes $(n)$ of PM6:Y6 devices with 10 and 45 wt \% donor are around 1.3, indicating that the recombination is primarily via the bimolecular pathways with a minor role of trap-assisted recombination. ${ }^{33,34}$ The slope of the dilute acceptor device (90 wt \% PM6) is very close to unity, which suggests that photogenerated carriers are purely lost via bimolecular recombination. Moreover, transient photovoltage (TPV) measurements were performed on PM6:Y6 solar cells, as shown in Figure 4c. The solar cells with 10 and 45 wt \% PM6 exhibit slower recombination with longer decay times of 49.1 and $75.5 \mu \mathrm{s}$, respectively; however, the device with $90 \mathrm{wt} \%$ PM6 shows much faster charge recombination with a shorter decay time of $31.6 \mu \mathrm{s}$, which agrees with the above results.
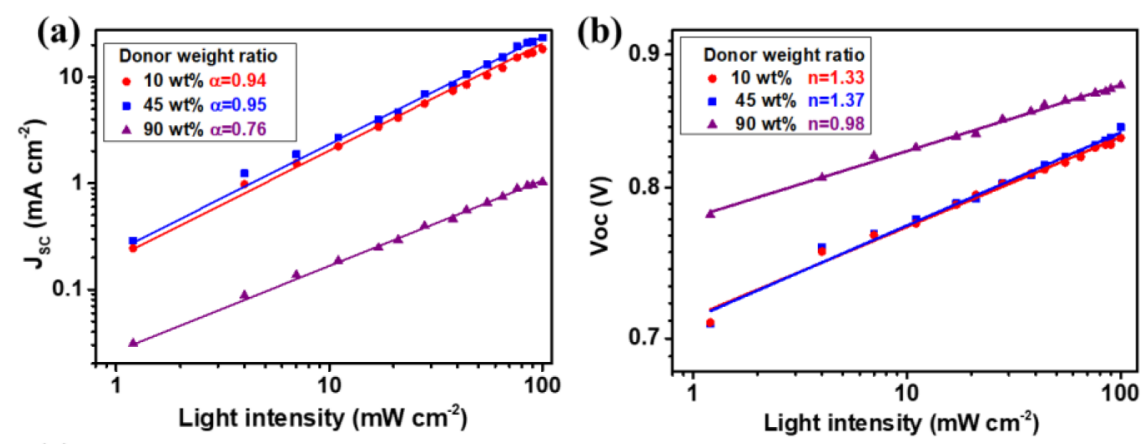

(c)

(d)
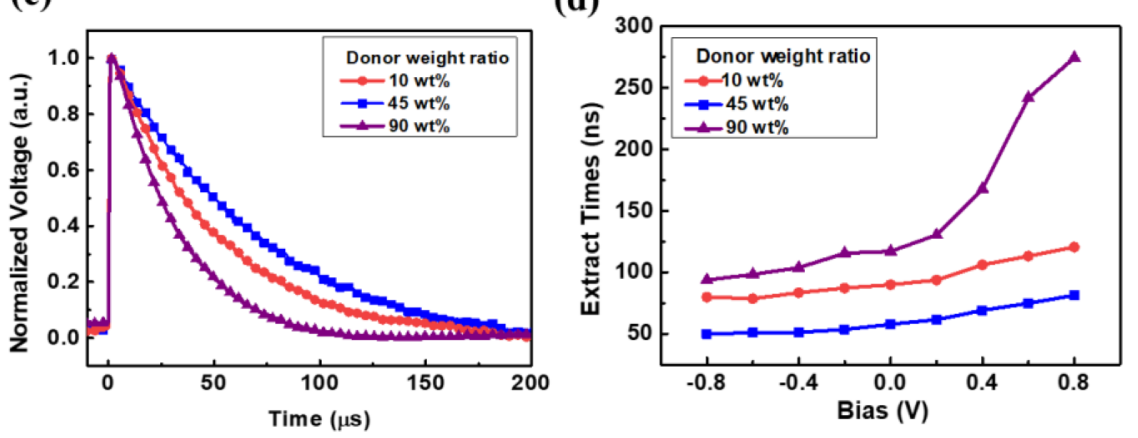

Figure 4. Light intensity-dependent (a) $J_{\mathrm{sc}}$ and (b) $V_{\text {oc }}$ characteristics of PM6:Y6 solar cells with various PM6 weight ratios. (c) TPV under opencircuit conditions. (d) Charge extraction time $\left(\tau_{\text {ext }}\right)$ as a function of external bias in the studied solar cells determined from TPC measurements. 
Table 1. Hole Mobilities of Pristine PM6, PS, and PMMA and PM6:Y6, PM6:PS, and PM6:PMMA BHJs with 10 wt \% PM6

\begin{tabular}{cccccccc} 
& PM6 & Y6 & PS & PMMA & PM6:Y6 & PM6:PS & PM6:PMMA \\
\hline$\mu_{\mathrm{h}}\left[\mathrm{cm}^{2} \mathrm{~V}^{-1} \mathrm{~s}^{-1}\right]$ & $3.2 \times 10^{-4}$ & $1.8 \times 10^{-4}$ & $9.2 \times 10^{-8}$ & $1.6 \times 10^{-8}$ & $6.8 \times 10^{-5}$ & $1.2 \times 10^{-4}$ & $3.7 \times 10^{-5}$ \\
\hline
\end{tabular}

In the case of extremely unbalanced $\mathrm{D} / \mathrm{A}$ ratios, a discontinuous interpenetrating network of donor and acceptor might be an important issue for charge transport. Also, a reduced contact area of anode/donor or cathode/acceptor could lead to a low charge extraction efficiency. Here we investigated charge extraction processes by using bias-dependent transient photocurrent (TPC) measurements (Figure S7); charge extraction time versus bias were obtained from TPC with a monoexponential decay model. In Figure 4d, the most notable difference is that, compared to the device with $90 \mathrm{wt} \%$ PM6, the solar cells with 10 and 45 wt \% PM6 contents show faster and nearly field-independent charge transport and extraction, especially near the low internal field region.

Our previous work demonstrated that charge extraction correlates with bias dependence of the external quantum efficiency (EQE) of OSCs. ${ }^{14}$ Bias-dependent EQE spectra of 10, 45, and 90 wt \% PM6-based devices were recorded and are shown in Figure S8. The EQE spectra of devices with 10 and 45 wt \% donor contents are almost insensitive to bias, which is consistent with the bias-TPC results. This feature can arise from the suppressed recombination loss at low internal fields, so that photogenerated carriers can be collected more efficiently, yielding high $J_{s c}$ and FF in the OSCs with $10 \mathrm{wt}$ \% PM6.

Above results demonstrate that the OSCs with 10 wt \% PM6 show high and balanced charge transport, which enables slow charge recombination and fast charge extraction. As for the origin of efficient hole transport in dilute donor devices, we assume following hole transport pathways: (i) There is possibility for holes tunneling between isolated donors. ${ }^{19}$ (ii) Holes can transport directly in donor phase. (iii) Y6 may facilitate the hole transport because it is an ambipolar conductor.

To clarity the hole transport mechanism in PM6:Y6 solar cells with 10 wt \% PM6, we compared the hole mobilities of PM6:Y6 with PM6:PS (polystyrene) with the same PM6 content because PS is an insulator with very low hole mobility in the order of $10^{-8} \mathrm{~cm}^{2} \mathrm{~V}^{-1} \mathrm{~s}^{-1}$. As for the results shown in Figure S9 and Table 1, the hole mobilities of PM6:Y6 and PM6:PS with 1:9 weight ratios are in the same order of magnitude, which are comparable to the hole mobility of pristine PM6, indicating the hole transport via the PM6 phase can be a dominant mechanism in 10 wt \% PM6 BHJs. To confirm this hypothesis, we measure the hole mobility of PM6 diluted by another insulator poly(methyl methacrylate) (PMMA). In Table 1, PM6:Y6 and PM6:PMMA blends with 10 wt \% PM6 also show comparable hole mobilities. Therefore, we conclude that PM6:Y6 blends even with $10 \mathrm{wt}$ \% PM6 can still maintain efficient hole transport. Furthermore, according to grazing-incidence wide-angle X-ray scattering (GIWAXS) data (Figure S10), the (100) diffraction peak for PM6 at $0.32 \AA^{-1}$ in in-plane was also observed in 10 wt \% PM6:Y6 films, which was most probably induced by the faceoriented Y6 molecules. ${ }^{35}$ This phenomenon reveals that, although in the dilute donor blend, the donor exhibits ordered structure, which can account for high hole mobility in dilute donor devices.
It is obvious that the dark $J-V$ curves of hole-only devices based on pristine $\mathrm{Y} 6$ exhibit a trap-filling process, but this phenomenon does not exist in PM6:Y6, PM6:PS, and PM6:PMMA blends (Figure S6 and Figure S9). In addition, hole transfer from donor to acceptor is generally unfavored, because the highest occupied molecular orbital (HOMO) level of Y6 $(-5.7 \mathrm{eV})$ is deeper than that of PM6 $(-5.48 \mathrm{eV})$, which was confirmed by cyclic voltammetry (CV) measurements (Figure S11 and Table S5). Therefore, it is plausible that NFA Y6 has little contribution to hole transport in the dilute donor structure with 10 wt \% PM6 (iii), even though Y6 has ambipolar transport characteristics. Given the discussions above, in the dilute donor devices, $10 \%$ of PM6 can form efficient hole transport pathways, enabling well-balanced charge transport with sufficiently high mobilities.

To conclude, the performance of PM6:Y6 solar cells with varying stoichiometries was comparatively studied. A high PCE exceeding $10 \%$ was achieved in the dilute donor devices $(10 \mathrm{wt}$ \% PM6) synergized by efficient hole transfer, charge transport, slow charge recombination, and field-insensitive extraction proved with results of TA, TPV, TPC, and bias-dependent EQE. Surprisingly, balanced electron and hole mobilities are achieved in the $10 \mathrm{wt} \%$ PM6 OSCs evaluated with SCLC in single carrier devices. By comparing the hole mobility of $10 \mathrm{wt}$ \% PM6 mixed with those of Y6, PS, or PMMA, we propose that the dominant hole transport in dilute PM6:Y6 blends is via the PM6 phase. Good performance of dilute donor heterojunctions may open new applications such as for nearinfrared photodetectors or semitransparent photovoltaic panels.

\section{ASSOCIATED CONTENT}

\section{SI Supporting Information}

The Supporting Information is available free of charge at https://pubs.acs.org/doi/10.1021/acs.jpclett.1c01219.

Materials, device fabrication, characterizations, calculation of HTE, J-V curves, PL, TA, and bias-EQE spectra, kinetics plots, AFM images, 2D GIWAXS patterns, CV, and tables of photovoltaic parameters, hole transfer kinetics, HTE, electron and hole mobilities, and redox potentials and energy levels (PDF)

\section{AUTHOR INFORMATION}

\section{Corresponding Authors}

Haiming Zhu - State Key Laboratory of Modern Optical Instrumentation, Center for Chemistry of High-Performance \& Novel Materials, Department of Chemistry, Zhejiang University, Hangzhou 310027, P. R. China; 이이.org/ 0000-0001-7747-9054; Email: hmzhu@zju.edu.cn

Yuan Zhang - School of Chemistry, Beijing Advanced Innovation Center for Biomedical Engineering, Beihang University, Beijing 100191, P. R. China; 이이.org/00000003-0670-2428; Email: yuanzhang@buaa.edu.cn

Fengling Zhang - Department of Physics, Chemistry and Biology (IFM), Linköping University, Linköping 58183, Sweden; @ orcid.org/0000-0002-1717-6307; Email: fengling.zhang@liu.se 


\section{Authors}

Nannan Yao - Department of Physics, Chemistry and Biology (IFM), Linköping University, Linköping 58183, Sweden

Jianqiu Wang - School of Chemistry, Beijing Advanced Innovation Center for Biomedical Engineering, Beihang University, Beijing 100191, P. R. China

Zeng Chen - State Key Laboratory of Modern Optical Instrumentation, Center for Chemistry of High-Performance \& Novel Materials, Department of Chemistry, Zhejiang University, Hangzhou 310027, P. R. China

Qingzhen Bian - Department of Physics, Chemistry and Biology (IFM), Linköping University, Linköping 58183, Sweden; orcid.org/0000-0001-5276-7172

Yuxin Xia - Institute for Materials Research (IMOIMOMEC), Hasselt University, 3590 Diepenbeek, Belgium

Rui Zhang - Department of Physics, Chemistry and Biology (IFM), Linköping University, Linköping 58183, Sweden

Jianqi Zhang - National Center for Nanoscience and

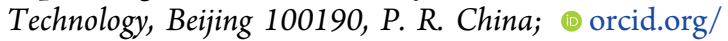
0000-0002-3549-1482

Leiqiang Qin - Department of Physics, Chemistry and Biology (IFM), Linköping University, Linköping 58183, Sweden; ○ orcid.org/0000-0002-7047-0927

Complete contact information is available at:

https://pubs.acs.org/10.1021/acs.jpclett.1c01219

\section{Notes}

The authors declare no competing financial interest.

\section{ACKNOWLEDGMENTS}

N.Y. and F.Z. acknowledge funding from Knut and Alice Wallenberg Foundation under contract 2016.0059, the Swedish Government Research Area in Materials Science on Functional Materials at Linköping University (Faculty Grant SFO-Mat-LiU No. 200900971) and China Scholarship Council (CSC) (No. 201708370115). Y.Z. thanks the financial support by the National Natural Science Foundation of China (No. 21875012, 21674006). H.Z. acknowledges the financial support by the National Key Research and Development Program of China (2017YFA0207700).

\section{REFERENCES}

(1) Lin, Y.; Wang, J.; Zhang, Z. G.; Bai, H.; Li, Y.; Zhu, D.; Zhan, X. An Electron Acceptor Challenging Fullerenes for Efficient Polymer Solar Cells. Adv. Mater. 2015, 27 (7), 1170-1174.

(2) Li, S.; Ye, L.; Zhao, W.; Zhang, S.; Mukherjee, S.; Ade, H.; Hou, J. Energy-Level Modulation of Small-Molecule Electron Acceptors to Achieve over 12\% Efficiency in Polymer Solar Cells. Adv. Mater. 2016, 28 (42), 9423-9429.

(3) Zhao, W.; Li, S.; Yao, H.; Zhang, S.; Zhang, Y.; Yang, B.; Hou, J. Molecular Optimization Enables over 13\% Efficiency in Organic Solar Cells. J. Am. Chem. Soc. 2017, 139 (21), 7148-7151.

(4) Hou, J.; Inganäs, O.; Friend, R. H.; Gao, F. Organic Solar Cells Based on Non-Fullerene Acceptors. Nat. Mater. 2018, 17 (2), 119128.

(5) Yuan, J.; Zhang, Y.; Zhou, L.; Zhang, C.; Lau, T. K.; Zhang, G.; Lu, X.; Yip, H. L.; So, S. K.; Beaupré, S.; et al. Fused Benzothiadiazole: A Building Block for n-Type Organic Acceptor to Achieve High-Performance Organic Solar Cells. Adv. Mater. 2019, 31 (17), 1807577.

(6) Yuan, J.; Zhang, Y.; Zhou, L.; Zhang, G.; Yip, H. L.; Lau, T. K.; Lu, X.; Zhu, C.; Peng, H.; Johnson, P. A.; et al. Single-Junction Organic Solar Cell with over 15\% Efficiency Using Fused-Ring
Acceptor with Electron-Deficient Core. Joule 2019, 3 (4), 11401151.

(7) Liu, Q.; Jiang, Y.; Jin, K.; Qin, J.; Xu, J.; Li, W.; Xiong, J.; Liu, J.; Xiao, Z.; Sun, K.; et al. 18\% Efficiency Organic Solar Cells. Sci. Bull. 2020, 65 (4), 272-275.

(8) Lin, Y.; Firdaus, Y.; Isikgor, F. H.; Nugraha, M. I.; Yengel, E.; Harrison, G. T.; Hallani, R.; El-Labban, A.; Faber, H.; Ma, C.; et al. Self-assembled Monolayer Enables Hole Transport Layer-free Organic Solar Cells with 18\% Efficiency and Improved Operational Stability. ACS Energy Lett. 2020, 5 (9), 2935-2944.

(9) Zhan, L.; Li, S.; Xia, X.; Li, Y.; Lu, X.; Zuo, L.; Shi, M.; Chen, H. Layer-by-layer Processed Ternary Organic Photovoltaics with Efficiency over 18\%. Adv. Mater. 2021, 33 (12), 2007231.

(10) Liu, J.; Chen, S.; Qian, D.; Gautam, B.; Yang, G.; Zhao, J.; Bergqvist, J.; Zhang, F.; Ma, W.; Ade, H.; et al. Fast Charge Separation in a Non-Fullerene Organic Solar Cell with a Small Driving Force. Nat. Energy 2016, 1, 16089.

(11) Li, S.; Zhan, L.; Sun, C.; Zhu, H.; Zhou, G.; Yang, W.; Shi, M.; Li, C.-Z.; Hou, J.; Li, Y.; et al. Highly Efficient Fullerene-Free Organic Solar Cells Operate at Near Zero Highest Occupied Molecular Orbital Offsets. J. Am. Chem. Soc. 2019, 141 (7), 3073-3082.

(12) Yuan, J.; Huang, T.; Cheng, P.; Zou, Y.; Zhang, H.; Yang, J. L.; Chang, S. Y.; Zhang, Z.; Huang, W.; Wang, R.; et al. Enabling Low Voltage Losses and High Photocurrent in Fullerene-Free Organic Photovoltaics. Nat. Commun. 2019, 10, 570.

(13) Bartesaghi, D.; Pérez, I. D. C.; Kniepert, J.; Roland, S.; Turbiez, M.; Neher, D.; Koster, L. J. A. Competition between Recombination and Extraction of Free Charges Determines the Fill Factor of Organic Solar Cells. Nat. Commun. 2015, 6, 7083.

(14) Wang, J.; Yao, N.; Zhang, D.; Zheng, Z.; Zhou, H.; Zhang, F.; Zhang, Y. Fast Field-Insensitive Charge Extraction Enables High Fill Factors in Polymer Solar Cells. ACS Appl. Mater. Interfaces 2020, 12 (34), 38460-38469.

(15) Zhang, J.; Tan, H. S.; Guo, X.; Facchetti, A.; Yan, H. Material Insights and Challenges for Non-Fullerene Organic Solar Cells Based on Small Molecular Acceptors. Nat. Energy 2018, 3, 720-731.

(16) Mihailetchi, V. D.; Wildeman, J.; Blom, P. W. M. Space-Charge Limited Photocurrent. Phys. Rev. Lett. 2005, 94, 126602.

(17) Jespersen, K. G.; Zhang, F.; Gadisa, A.; Sundström, V.; Yartsev, A.; Inganäs, O. Charge Formation and Transport in BulkHeterojunction Solar Cells Based on Alternating Polyfluorene Copolymers Blended with Fullerenes. Org. Electron. 2006, 7 (4), 235-242.

(18) Albes, T.; Xu, L.; Wang, J.; Hsu, J. W. P.; Gagliardi, A. Origin of Photocurrent in Fullerene-Based Solar Cells. J. Phys. Chem. C 2018, 122 (27), 15140-15148.

(19) Melianas, A.; Pranculis, V.; Spoltore, D.; Benduhn, J.; Inganäs, O.; Gulbinas, V.; Vandewal, K.; Kemerink, M. Charge Transport in Pure and Mixed Phases in Organic Solar Cells. Adv. Energy Mater. 2017, 7 (20), 1700888.

(20) Spoltore, D.; Hofacker, A.; Benduhn, J.; Ullbrich, S.; Nyman, M.; Zeika, O.; Schellhammer, S.; Fan, Y.; Ramirez, I.; Barlow, S.; et al. Hole Transport in Low-Donor-Content Organic Solar Cells. J. Phys. Chem. Lett. 2018, 9, 5496-5501.

(21) Tuladhar, S. M.; Poplavskyy, D.; Choulis, S. A.; Durrant, J. R.; Bradley, D. D. C.; Nelson, J. Ambipolar Charge Transport in Films of Methanofullerene and Poly(Phenylenevinylene)/Methanofullerene Blends. Adv. Funct. Mater. 2005, 15, 1171-1182.

(22) Guo, X.; Zhang, M.; Tan, J.; Zhang, S.; Huo, L.; Hu, W.; Li, Y.; Hou, J. Influence of D/A Ratio on Photovoltaic Performance of a Highly Efficient Polymer Solar Cell System. Adv. Mater. 2012, 24, 6536-6541.

(23) Karki, A.; Vollbrecht, J.; Dixon, A. L.; Schopp, N.; Schrock, M.; Reddy, G. N. M.; Nguyen, T. Understanding the High Performance of over 15\% Efficiency in Single-Junction Bulk Heterojunction Organic Solar Cells. Adv. Mater. 2019, 31, 1903868.

(24) Zhang, G.; Chen, X. K.; Xiao, J.; Chow, P. C. Y.; Ren, M.; Kupgan, G.; Jiao, X.; Chan, C. C. S.; Du, X.; Xia, R.; et al. Delocalization of Exciton and Electron Wavefunction in Non- 
Fullerene Acceptor Molecules Enables Efficient Organic Solar Cells. Nat. Commun. 2020, 11, 3943.

(25) Chen, Z.; Chen, X.; Qiu, B.; Zhou, G.; Jia, Z.; Tao, W.; Li, Y.; Yang, Y. M.; Zhu, H. Ultrafast Hole Transfer and Carrier Transport Controlled by Nanoscale-Phase Morphology in Nonfullerene Organic Solar Cells. J. Phys. Chem. Lett. 2020, 11 (9), 3226-3233.

(26) Qiu, B.; Chen, Z.; Qin, S.; Yao, J.; Huang, W.; Meng, L.; Zhu, H.; Yang, Y.; Zhang, Z. G.; Li, Y. Highly Efficient All-Small-Molecule Organic Solar Cells with Appropriate Active Layer Morphology by Side Chain Engineering of Donor Molecules and Thermal Annealing. Adv. Mater. 2020, 32 (21), 1908373.

(27) Chen, Z.; Chen, X.; Jia, Z.; Zhou, G.; Xu, J.; Wu, Y.; Xia, X.; Li, X.; Zhang, X.; Deng, C.; et al. Triplet Exciton Formation for Nonradiative Voltage Loss in High-efficiency Nonfullerene Organic Solar Cells. Joule 2021, DOI: 10.1016/j.joule.2021.04.002.

(28) Wen, T. J.; Liu, Z. X.; Chen, Z.; Zhou, J.; Shen, Z.; Xiao, Y.; Lu, X.; Xie, Z.; Zhu, H.; Li, C. Z.; et al. Simple Non-Fused Electron Acceptors Leading to Efficient Organic Photovoltaics. Angew. Chem., Int. Ed. 2021, DOI: 10.1002/anie.202101867.

(29) Brédas, J. L.; Sargent, E. H.; Scholes, G. D. Photovoltaic Concepts Inspired by Coherence Effects in Photosynthetic Systems. Nat. Mater. 2017, 16, 35-44.

(30) Bian, Q.; Ma, F.; Chen, S.; Wei, Q.; Su, X.; Buyanova, I. A.; Chen, W. M.; Ponseca, C. S.; Linares, M.; Karki, K. J.; et al. Vibronic Coherence Contributes to Photocurrent Generation in Organic Semiconductor Heterojunction Diodes. Nat. Commun. 2020, 11, 617.

(31) Huo, Y.; Gong, X. T.; Lau, T. K.; Xiao, T.; Yan, C.; Lu, X.; Lu, G.; Zhan, X.; Zhang, H. L. Dual-accepting-unit Design of Donor Material for All-small-molecule Organic Solar Cells with Efficiency Approaching 11\%. Chem. Mater. 2018, 30, 8661-8668.

(32) Koster, L. J. A.; Mihailetchi, V. D.; Xie, H.; Blom, P. W. M. Origin of the Light Intensity Dependence of the Short-Circuit Current of Polymer/Fullerene Solar Cells. Appl. Phys. Lett. 2005, 87 (20), 203502.

(33) Koster, L. J. A.; Mihailetchi, V. D.; Ramaker, R.; Blom, P. W. M. Light Intensity Dependence of Open-Circuit Voltage of Polymer:Fullerene Solar Cells. Appl. Phys. Lett. 2005, 86 (12), 123509.

(34) Cowan, S. R.; Roy, A.; Heeger, A. J. Recombination in Polymerfullerene Bulk Heterojunction Solar Cells. Phys. Rev. B: Condens. Matter Mater. Phys. 2010, 82, 245207.

(35) Zhu, L.; Zhang, M.; Zhou, G.; Hao, T.; Xu, J.; Wang, J.; Qiu, C.; Prine, N.; Ali, J.; Feng, W.; et al. Efficient Organic Solar Cell with $16.88 \%$ Efficiency Enabled by Refined Acceptor Crystallization and Morphology with Improved Charge Transfer and Transport Properties. Adv. Energy Mater. 2020, 10 (18), 1904234. 